\title{
Evaluation of Basophil Infiltration into the Skin Lesions of Tick Bites
}

\author{
Kyoko Nakahigashi Atsushi Otsuka Kaori Tomari Yoshiki Miyachi \\ Kenji Kabashima \\ Department of Dermatology, Kyoto University Graduate School of Medicine, Kyoto, Japan
}

\section{Key Words}

Basophil · Tick bites

\section{Abstract}

Recently, it has been described that basophils play an essential role in antibody-mediated acquired immunity against ticks in mice. However, it is still unknown whether basophil infiltration has any significance in the infestation with ticks in humans. In this report, we have evaluated the infiltration of basophils into human skin lesions of tick bites.

\section{Introduction}

Basophils constitute less than $1 \%$ of blood leukocytes and their pathophysiological roles in human allergic reactions remain mostly unclear, although basophil infiltration into the skin has been demonstrated in some human skin diseases [1-3]. Recent studies have demonstrated that basophils play a critical role in the development of IgE-mediated chronic allergic reactions by functioning as an initiator in mice [4, 5]; basophils also promote $\mathrm{T}$ helper (Th) 2 skewing by antigen presentation in helminthic infections and in response to protease allergens in an IgE-independent manner in mice [6, 7]. Additionally, a recent study has interestingly demonstrated that basophils play an essential role in antibody-mediated acquired immunity against ticks in mice [8]. However, it remains unknown whether basophil infiltration might be involved in the infestation of ticks in humans. Herein, we have evaluated the infiltration of basophils into human skin lesions of tick bites. 


\section{Case Presentation}

During a 3-year period (2009-2011), we have examined 8 patients with tick bites (3 males and 5 females, mean age \pm standard deviation [SD] $41.5 \pm 17.1$ years, range 18-62). Skin biopsies from skin lesions with tick bites were examined by hematoxylin-eosin staining and immunostaining. Staining for basophils were incubated with anti-human basophil antibody (2D7) (BioLegend, San Diego, Calif., USA), followed by incubation with biotinylated horse anti-mouse serum and then with avidin-biotin-peroxidase complex (ABC-AP; Vector Laboratories, Burlingame, Calif., USA). Coloring reaction was performed with alkaline phosphatase substrate kit I (Vector). This study was carried out in accordance with the Declaration of Helsinki and was approved by the ethics committee of Kyoto University, Japan. Informed consent was obtained from all patients enrolled in the study. Eosin-positive binuclear cells and 2D7-immunoreactive cells represent eosinophils and basophils, respectively (fig. 1a, b). Basophils were observed to infiltrate into the skin lesions of 3 patients, but not in the other 5 patients. The numbers of infiltrating eosinophils and basophils were enumerated in 5 high-power fields (table 1). There was no significant correlation between the numbers of eosinophils and basophils infiltrating into the skin.

\section{Discussion}

In this report, we have evaluated the presence of basophils in human skin lesions of tick bites. Basophil infiltration into the skin is considered to be accompanied by eosinophil infiltration [1]. Intriguingly, we found that some cases did not exhibit basophil infiltration in spite of the infiltration of eosinophils. In addition, the number of basophils in the skin and the ratio of tissue basophils/eosinophils varies among skin diseases. The ratio of basophils/eosinophils is approximately 0.1 in atopic dermatitis, 1 in prurigo, long-lasting urticaria, bullous pemphigoid and eosinophilic pustular folliculitis, and greater than 1 in Henoch-Schönlein purpura [1]. In our study, the average ratio \pm SD was $0.06 \pm 0.09$, which is quite a low value compared to other skin diseases. Previously, Ito et al. [1] reported the low value of 0.1 in atopic dermatitis in their study could be due to biased cytokine milieu toward Th1 in chronic skin lesions. It was also reported that the infiltration of basophils at tickfeeding sites of mice occurred during the second, but not the first tick infestation [8]. Considering these findings, the difference in our study may or may not be derived from the differences between the first infestation. Although the number of patients in this study is limited, the absence or presence of basophils in tick bite lesions suggest that basophils might be involved in the development of acquired resistance to tick bites. Further investigations might shed a light on the protection from tick bites.

\section{Disclosure Statement}

The authors declare no conflict of interest. 


\section{References}

1 Ito Y, Satoh T, Takayama K, Miyagishi C, Walls AF, Yokozeki H: Basophil recruitment and activation in inflammatory skin diseases. Allergy 2011;66:1107-1113.

-2 Otsuka A, Miyagawa-Hayashino A, Walls A, Miyachi Y, Kabashima K: Comparison of basophil infiltration into the skin between eosinophilic pustular folliculitis and neutrophilic folliculitis. J Eur Acad Dermatol Venereol 2012;26:527-529.

-3 Otsuka A, Ozaki M, Horiguchi Y, Murata Y, Kumano K, Nogami R, Goto M, Walls AF, Ishii N, Miyachi Y, Kabashima K: Basophils infiltrate the skin lesions in lepromatous leprosy. Acta Derm Venereol 2013;93:8889.

-4 Mukai K, Matsuoka K, Taya C, Suzuki H, Yokozeki H, Nishioka K, Hirokawa K, Etori M, Yamashita M, Kubota T, Minegishi Y, Yonekawa H, Karasuyama H: Basophils play a critical role in the development of IgEmediated chronic allergic inflammation independently of T cells and mast cells. Immunity 2005;23:191202.

5 Obata K, Mukai K, Tsujimura Y, Ishiwata K, Kawano Y, Minegishi Y, Watanabe N, Karasuyama H: Basophils are essential initiators of a novel type of chronic allergic inflammation. Blood 2007;110:913-920.

-6 Yoshimoto T, Yasuda K, Tanaka H, Nakahira M, Imai Y, Fujimori Y, Nakanishi K: Basophils contribute to $\mathrm{T}(\mathrm{H}) 2-\mathrm{IgE}$ responses in vivo via IL-4 production and presentation of peptide-MHC class II complexes to CD4+ T cells. Nat Immunol 2009;10:706-712.

7 Sokol CL, Chu NQ, Yu S, Nish SA, Laufer TM, Medzhitov R: Basophils function as antigen-presenting cells for an allergen-induced T helper type 2 response. Nat Immunol 2009;10:713-720.

-8 Wada T, Ishiwata K, Koseki H, Ishikura T, Ugajin T, Ohnuma N, Obata K, Ishikawa R, Yoshikawa S, Mukai K, Kawano Y, Minegishi Y, Yokozeki H, Watanabe N, Karasuyama H: Selective ablation of basophils in mice reveals their nonredundant role in acquired immunity against ticks. J Clin Invest 2010;120:2867-2875.

Table 1. The numbers of infiltrating basophils and eosinophils in tick bite lesions

\begin{tabular}{llllll}
\hline Case & Age & Sex & Basophils/HPF (B) & Eosinophils/HPF (E) & Ratio (B/E) \\
\hline 1 & 18 & F & 0 & 12 & 0 \\
2 & 22 & F & 0 & 2 & 0 \\
3 & 28 & F & 0 & 12 & 0 \\
4 & 41 & M & 1 & 11 & 0.09 \\
5 & 49 & M & 2 & 9 & 0.23 \\
6 & 52 & F & 4 & 22 & 0.18 \\
7 & 60 & M & 0 & 23 & 0 \\
8 & 62 & F & 0 & $11.5 \pm 7.8$ & 0 \\
\hline Mean \pm SD & $41.5 \pm 17.1$ & 3 M, 5 F & $0.88 \pm 1.4$ & $0.06 \pm 0.09$ \\
\hline
\end{tabular}




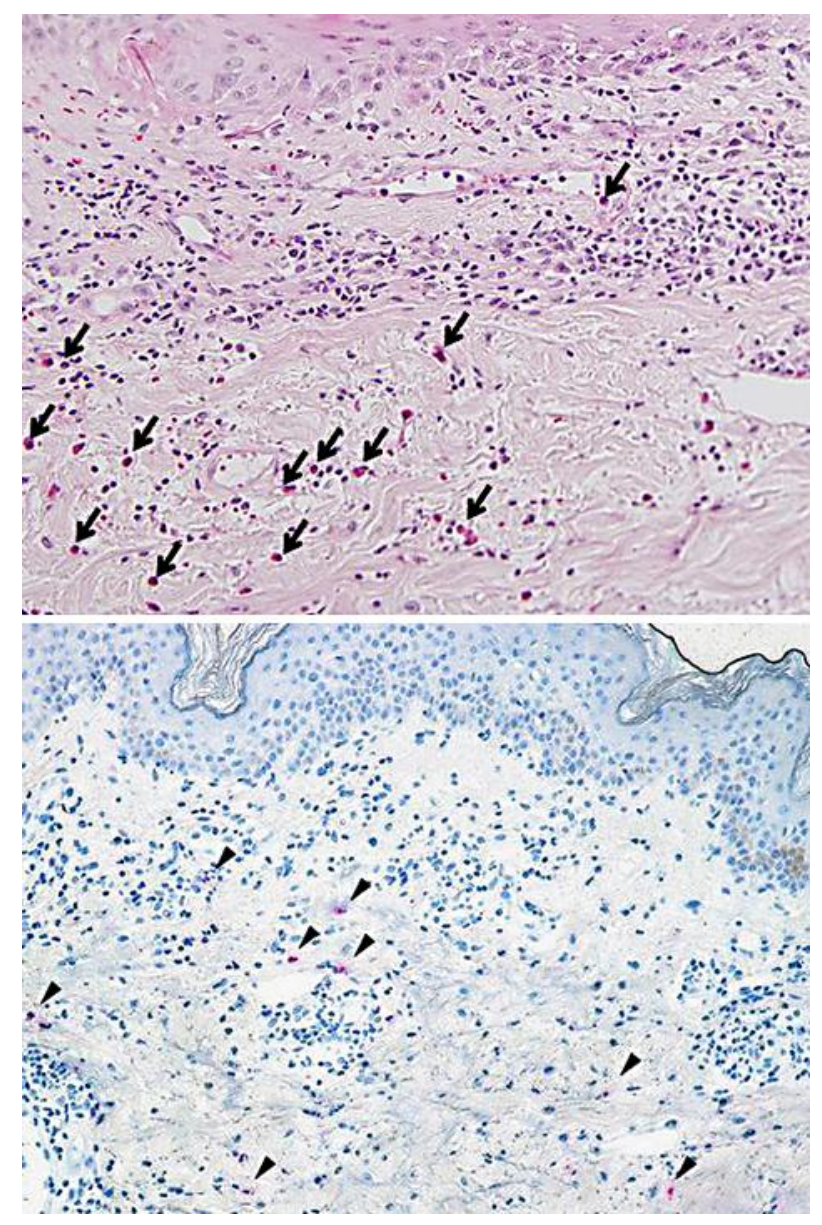

Fig. 1. Histology of the lesion with tick bites of a representative case, a 52-year-old healthy woman. Hematoxylin-eosin staining showed the infiltration of eosinophils (arrows). Immunostaining of 2D7 antibody indicated the infiltration of basophils (arrowheads). 